\title{
Design of Ergonomic Illumination Systems for Cultural, Medical, Educational Facilities
}

\author{
Nikolai Bogdanov, Dmitry Zhdanov, Igor Potemin, Andrey Zhdanov \\ Visualization Technologies Department University ITMO Saint-Petersburg, Russia
}

How to cite this paper: Bogdanov, N., Zhdanov, D., Potemin, I., \& Zhdanov, A. (2017). Design of Ergonomic Illumination Systems for Cultural, Medical, Educational Facilities. The Educational Review, USA, 1(4), 85-90.

http://dx.doi.org/10.26855/er.2017.04.001

Corresponding author: Nikolai Bogdanov, Visualization Technologies Department University ITMO Saint-Petersburg, Russia.

\begin{abstract}
LED panels with light-guiding plates can be good substitution of traditional lighting fixtures in museums. They can provide high quality of comfortable general lighting at public places. This article describes design of LED light fixtures for cultural, medical, educational facilities, where applied strict requirements for illumination. Authors made optical design of round light-guide plate with microstructures, which is component of LED panel, and developed solution technology for producing light-guide plate by $\mathrm{CO}_{2}$ laser machine-tool, and got sufficient results by comparing simulation model of lightguide plate and produced light-guide plate.
\end{abstract}

\section{Keywords}

LED lighting fixtures, light-guiding plates, design and producing, ergonomics, microstructures

\section{Introduction}

Today in Russia there is a governmental program (The resolution N 898, The resolution N 275), which is directed to replacement non-efficient lighting fixtures, like incandescent lamps luminescence lamps, mercury-vapor lamps and other gas-discharge lamps. All public utility companies, for example, educational facilities, medical facilities, cultural facilities should apply LED lighting fixtures. That light fixtures have got good effectiveness, can save energy consumption and money, therefore, the market of LED lighting is growing day by day.

A typical light fixture consists of PCBs with LEDs, driver, body frame (heater), diffuser of light or lenses, reflectors for LEDs, commutation wires. The most popular kind of LED light fixtures, which used in most cases, is office LED light fixtures. That light fixtures usually have sizes such as $600 \mathrm{~mm}$ x $600 \mathrm{~mm}, 300 \mathrm{~mm} \times 600 \mathrm{~mm}, 1200 \mathrm{~mm} \times 300 \mathrm{~mm}$ and etc. There is a variety of office LED light fixtures, offered in our country, outstanding factories make LED light fixtures with different characteristics, provide high quality production and warrant reliable rate. On the other hand, there are small manufacturers, that make cheaper light fixtures, but the quality of production in many cases is not good, that can influence people health, especially vision.

There are several important parameters of light fixture-overall luminance $\left(\mathrm{cd} / \mathrm{m}^{2}\right)$ of light fixture and equability of luminance, expressed by maximum and minimum luminance ratio of emitting surface. Human eyes react to these parameters. LEDs have very high lumen efficiency and compact sizes, during the operating, high luminance of LEDs may cause discomfort of eyes. Certainly, for light fixtures, which used in public places, are applied different kinds of diffusers of light: matte, "crushed ice", "prismatic", "pearls", but sometimes it does not enough for the implementation requirements. Therefore, in spite of energy efficiency, it should not be forgotten about comfort. Not all LED light 
fixtures could be used in schools, hospitals or museums, requirements for lighting are very strict (Onischenko,2012).

Trends of development light fixtures shows, that energy efficient is improved, and energy consumption is decrease. That parameters are provided by applying high energy efficient LEDs with high luminance. Therefore, if high energy efficient LEDs will use in traditional construction of LED light fixtures, it will be difficult to say about ergonomics. Actually, there is a problem, how can provide low energy consumption of light fixture without discomfort for human's eyes.

There are varieties of suitable technical solutions - one of them LED light fixtures with backlight modules (LED panels with BLM). The basis of this device is phenomena of propagation light in optical medium by the law of total internal reflection and scattering at the microstructures. Due to special position of LEDs on the end-face of light-guiding plate with microstructures and diffuser sheet, light scattering is provided and such kind of light distribution is more comfortable than direct lighting. Principle of performance of LED panels was kept from LCD displays, where lightguiding plates and backlight modules are applied for a long time.

Domestic market offers varieties of LED panels, but most of them are being produced in China and other countries. There are many reasons, why LED panels are not produced in our country. One of them connected with absence of technologies for light-guiding plates production. The authors have great experience in optical design different lightguiding plates and illumination systems (Zhdanov, 2013; Garbul, 2014; Zhdanov, 2014; Sokolov, 2014; Zhdanov, 2015; Zhdanov, 2016). But for complex development LED panels, especially light-guiding plates, besides designing its need to develop manufacturing technology. At the beginning of research, review of technologies for manufacturing light-guiding plates and forming microstructures was prepared. Authors made a suggestion about application laser machine tool for manufacturing light-guiding plate. For experiments, round LED panel with round light-guiding plates were chosen.

\section{Principle of Performance Light-guiding Plates}

The main physical phenomenon of light-guiding plate is light scattering at microstructures is shown on Fig.1 Light from LED is directed to end-face of light-guiding plate, after entrance in plate light is propagate in optical medium due to internal total reflection. For remove light from light-guiding plate, it is necessary that surface has microstructures (dimples). When light is achieved microstructures, it is scattered and directed to output surface (Zhdanov, 2016).

For achievement equal level of luminance at whole emitting surface of light-guiding plate, it is necessary to create especial density distribution of dimples. For defining optimal distribution of microstructures, it is need to simulate light fixture and make optical design for calculate luminance.

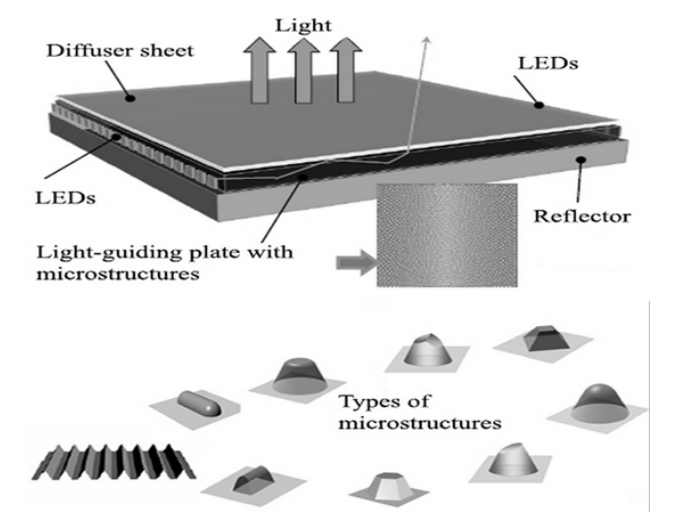

Fig.1. Light propagation in light-guiding plate and types of microstructures. 


\section{Selection Operating Mode of Laser Machine Tool}

Before modelling light fixture, we investigated technical opportunities of laser machine tool for forming hemispheres microstructures at plexiglas.

We tested several modes of power level and movement speed of laser had. Laser machine tool has got $\mathrm{CO}_{2}-$ laser tube, power of tube was $40 \mathrm{~W}$, this equipment could provide engraving and cutting plexiglas, wood, plastic and another nonmetallic material (www.gccworld.com). Connection with laser machine tool was provide with PC, the software for setting parameters of levels of speed and power was Corel Draw. This software is oriented for working with raster images and vector images, and it has a software plugin for control laser machine tool. Dimples shape was set as hemisphere, we chosen between different sizes from $500 \mu \mathrm{m}$ to $1000 \mu \mathrm{m}$. Dimples was formed at small areas. After laser engraving, we observed shape and relief for every test dimples by microscope, during observing we measured overall dimensions of dimples. It was revealed, that at high energy levels, dimples have cylindrical shape, not semi spheres. In addition, at high level of power energy, we observed a lot of products of combustion.

During selection optimal speed of movement laser had, we observed, that dimple's cross section of shape was changing from round to elliptical when speed was increased.

For choosing optimal operating mode, it was performed several dozen tests. However, ideal dimple shape was not achieved: formed microstructures were similar with elliptical, but radii was differed by 10 percent. Also, formed dimples have random air bubbles, and about seven-ten grooves were at one dimple.

Finally, we determined that for achieve optimal shape of dimples with diameter of cross section equal $700 \mu \mathrm{m}$, it is need to set laser power as $14 \mathrm{~W}$, and to set speed of laser had as $2 \mathrm{~m} / \mathrm{sec}$.

The best parameters of dimples, with random air bubbles and grooves, were applied in the computer model.

\section{Modelling and Optical Calculations}

Optical calculation was done in program complex Lumicept (www.integra.jp). For making physical correct model of light fixture, we provide next operations. At first, we made equidistant distribution of dimples and fabricate light-guiding plate at machine tool. We used special method of forming local-equidistant distribution of microstructures, which exclude moire (Zhdanov, 2015). In this method, local scattering properties are achieved due to variation local densities of repeated scattered microelements, and distribution periodicity is reduced by local variation coordinates of scattering microstructures.

The second step consists of, that we made calculation with equidistant distribution of dimples, and made some adjustments, in order to model of light fixture will be similar with real light fixtures. During making settings in computer mode, end-face of light-guiding plate was set as real, with some irregularity of surface and small inclination. So, we set optical properties not only at light-guiding plate and dimples, but also at light diffuser and at body frame surface.

Therefore, we could trust for correctness our model. At third, it was performed optimization of dimple distribution for achievement equitability of luminance. When dimple distribution had been received, new light-guiding plate was produced by laser machine tool.

\section{Producing Prototype Model}

We have got a production model of round LED light panel, which consist of light-guiding plate, LEDs, driver, body frame (heater), diffuser of light, and reflector. This LED panel is import production, but we could compare our prototype of light-guiding plate with another one in identical conditions, in the same construction of LED panel. Plexiglas was chosen by the way of optical material. Light-guiding plate was round, diameter was $257.5 \mathrm{~mm}$, thickness is $4 \mathrm{~mm}$. 
Dimple was a segment of sphere are shown on Fig.2, diameter of segment was $700 \mathrm{mkm}$, height was $12 \mathrm{mkm}$.

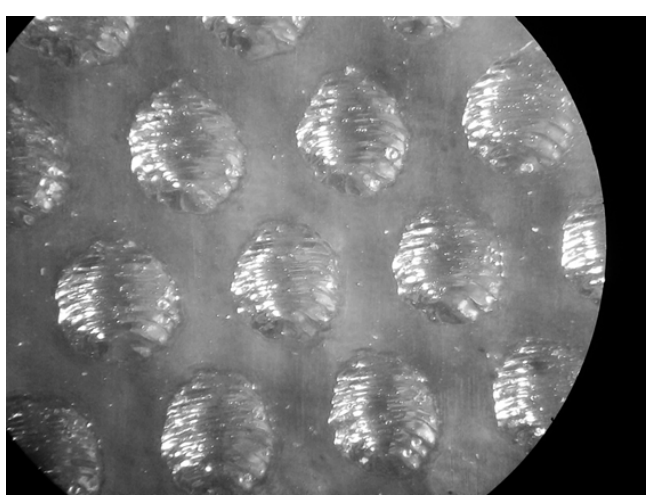

Fig.2. Microstructures.

Producing of light-guiding plate consists of two steps: the first was forming microstructures; the second was cutting of material.

We applied laser for material processing without oxygen stream, because it helped to avoid spraying of combustion products around plate, and around microstructures. Products of combustion have such negative impacts on the light scattering.

One of the positive features of laser cutting is producing transparent and smooth end-face, where input light has small losses.

Time for producing one prototype of light guiding plate was 40 minutes, process is shown on Fig.3. One the one hand, this time for producing prototype is normal, but on the other hand, for large manufacturing it is very slowly.

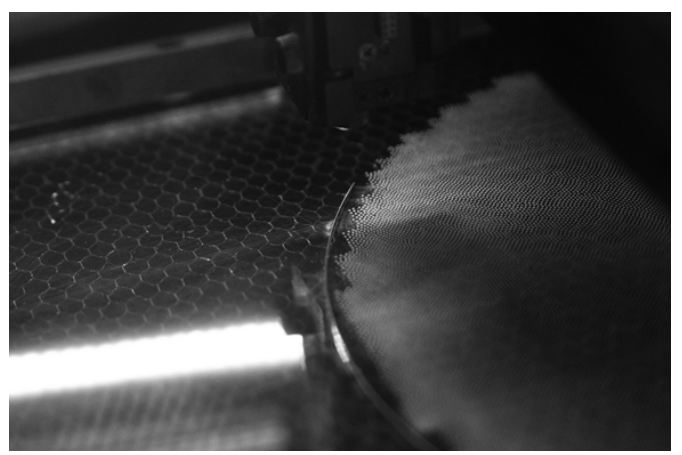

Fig.3. Process of producing light-guiding plate.

The main advantage of suggested producing technology is absence very high expensive equipment, such for punching or molding. Suggested technology is very convenient for producing small batch of lighting fixtures. If a museum, or another public place, needed individual lighting fixtures such type, it will be able to producing with this technology.

\section{Measuring and Results}

Produced prototype was set in light fixture body frame, after that measuring of lighting and electrical parameters was provide in photometric laboratory JSC «Svetlana-LED» (www.soptel.ru), so original light fixture with its own light guiding plate was measured too. We compared parameters both light-guiding plates. We measured luminance of emitting 
surface by different direction with luminance meter Konica Minolta. For measuring luminous flux and luminous intensity distribution curve we used goniophotometer system "Everfine", results are shown on Fig.4. After measuring lighting parameters, to see luminance distribution, exactly bright and dark areas, we made photos with camera Nikon D90, shutter speed was at level 1/4000 sec, diaphragm was at level $\mathrm{F}=10$, photos are shown on Fig.5. We explored photos with luminance variance, and analyzed quality of producing. We observed areas with different luminance, after that identified the reasons for the difference calculation.

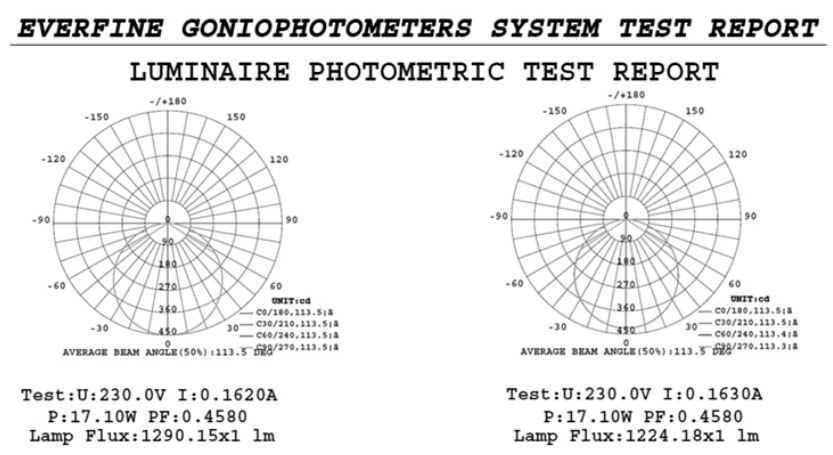

Fig.4. Luminaire photometric test report. Original light-guiding plate (left), produced prototype (right).

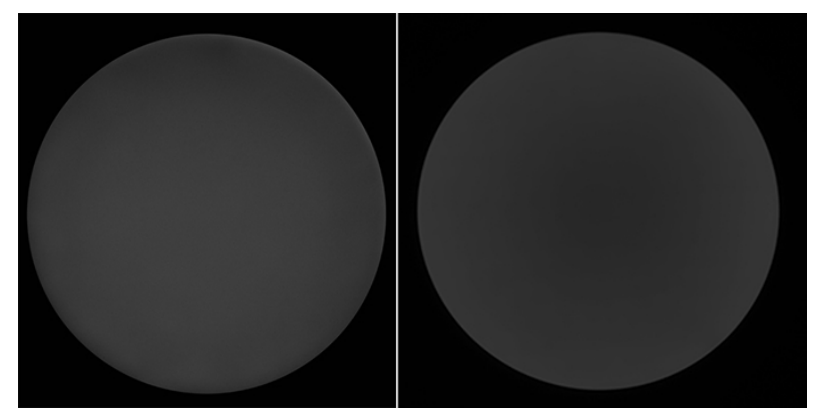

Fig.5. Photos of emitting surfaces of LED panel. Original light-guiding plate (left), produced prototype (right).

\section{Conclusion}

Authors can achieve good results in developing simple technology for producing light guiding plates with microstructures. By comparison produced prototype light-guiding plate with serial light-guiding plate, it was found, that light flux is differ by 5\%,1224 $\mathrm{lm}$ has produced prototype, $1290 \mathrm{~lm}$ has another one. Measurement uncertainty of goniophotometer system is $5 \%$ too. Ratio of luminance maximum and luminance minimum produced prototype light guiding plate was less than $10 \%$, ratio of serial light-guiding plate was $30 \%$, results are shown on Fig.6. Obtained results is differing from calculation model, this could be caused by inaccurate data of optical parameters of plexiglas, and geometrical parameters of dimples.

Applying suggested technology can solve individual tasks in projects of illumination museums, social and public places. Applying laser machine tool is adaptable approach for producing light guiding plates, and allow to producing different forms. Results of measurement producing light-guiding plate are just as well as serial light-fixture. This technology has good prospect, it can apply not only for producing light fixtures, but also for producing light-technics, based on lightguiding plates. 


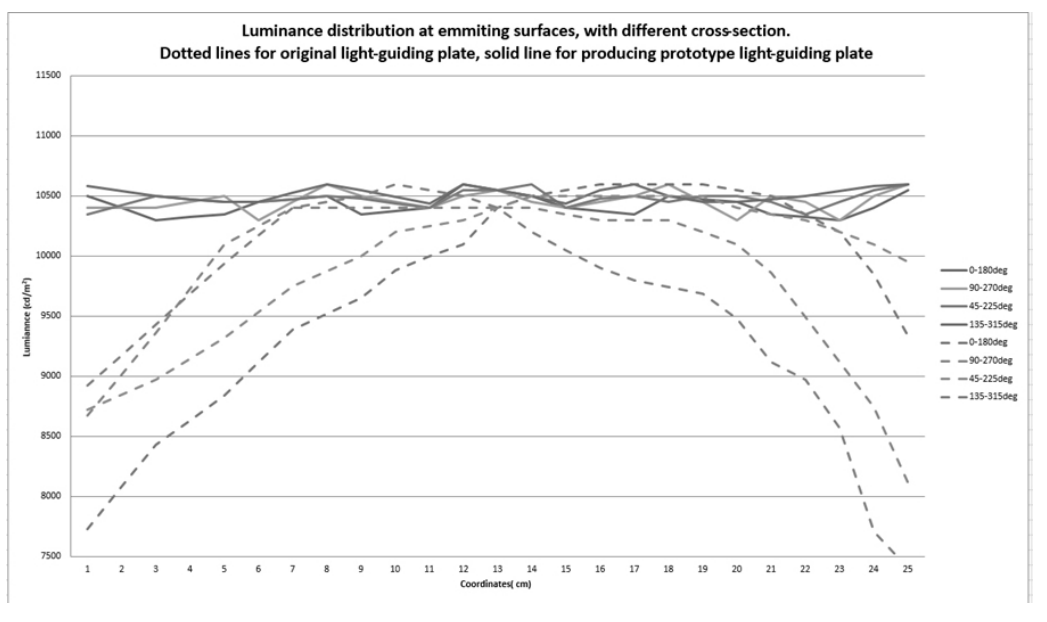

Fig.6. Luminance distribution at emitting surfaces.

\section{References}

Garbul, A. A. Garbul, D., Zhdanov, I. S., Potemin, V. G., Sokolov. (2014). Application of Methods of Bi-directional Stochastic Ray Tracing for Light Analysis and Lighting Design", XI International Conference "Applied Optics-2014": The Conference Proceedings.

Onischenko. (2012). The Official Letter from G.G. Onischenko N 01/11157-12-32 from 01.10.2012. On the Organization of Sanitary Supervision over the Use of Energy Saving Light Sources.

Sokolov, V. G., Sokolov, D. D., Zhdanov, I. S., Potemin, A. G., Voloboy, V. A., Galaktionov, N., Kirilov. (2014). Modeling and Computer Design of Liquid Crystal Display Backlight with Light Polarization Film. Optical Review, IET, 21(5), 642-650.

The Resolution N 898 - The Resolution of the Government of the Russian Federation from 28th August 2015 г. N 898.

The Resolution N 275 - The Resolution of the Government of the Russian Federation from 7th March 2017 г. N 275.

Zhdanov, D., Zhdanov, A. A., Garbul, V., Mayorov, V. G., Sokolov, I. S., Potemin, T., Hyodo, A. G., Voloboy, V. A., Galaktionov. (2013). Automatic Design of Illumination Systems. Optical Review, IET, 20(2), 155-159.

Zhdanov, D., Zhdanov, A. A., Garbul, , I. S., Potemin, V. G., Sokolov. (2014). Modeling and Optimization of Lighting Systems with Scattering Microstructures on a Curved Surface XI International Conference "Applied Optics-2014”: The Conference Proceedings-T. 2.

Zhdanov, D. D., Zhdanov, A. D., Zhdanov, I. S., Potemin, V. Y., Venediktov, V. A., Galaktionov. (2015). A Method of Fast Building of Locally Equidistant Distributions of Geometrical Objects of Illumination Systems. Optics and Spectroscopy, IET, 119(2), $311-317$.

Zhdanov, D. D., Zhdanov, A. D., Zhdanov, I. S., Potemin, V. Y., Venediktov, V. A., Galaktionov. (2015). A Quick Method of Constructing Locally Equidistant Distributions of Geometric Objects Lighting Systems . Optics and Spectroscopy, 119(2), $329-336$.

Zhdanov, D. D., Zhdanov, I. S., Potemin, N., Bogdanov, A., Voloboy, A., Garbul, V., Sokolov, V., Galaktionov. (2016). Computation Model of the Physically Accurate Simulation of the Light Transformation on the Free form Surfaces with Microelements. EOSAM 2016, IET, 2, S04. 